\title{
Morfología y Morfometría del Disco de la Articulación Témporomandibular en Fetos y Adultos Humanos
}

\author{
Morphology and Morphometry of the Temporomandibular Joint Disc in Human Fetus and Adults \\ "Fernando Matamala Vargas; "* Ramón Fuentes Fernández \& **'Mónica Ceballos Casanova
}

MATAMALA, V. F.; FUENTES, F. R. \& CEBALLOS, C. M. Morfología y morfometría del disco de la articulación témporomandibular en fetos y adultos humanos. Int. J. Morphol., 24(2):245-250, 2006.

RESUMEN: La articulación témporomandibular (ATM) humana, sinovial y bicondílea, es un conjunto de estructuras que permite la relación anatómica y funcional entre el hueso temporal y la mandíbula. Esta articulación permite realizar los movimientos mandibulares y las funciones del sistema estomatognático. Uno de los elementos de la ATM es el disco articular, que divide a la articulación en dos compartimientos y relaciona el proceso condilar con la fosa mandibular y con la eminencia articular del hueso temporal. Con el propósito de obtener información de la morfometría del disco articular, se analizaron y midieron 10 discos articulares de individuos adultos, de una edad promedio de 60 años, y 10 discos articulares de fetos con más de 28 semanas de gestación.

La forma del disco articular de la ATM de adultos corresponde a las descripciones de la literatura y el disco articular del feto muestra una zona retrodiscal poco desarrollada. El promedio de las medidas del disco articular de la ATM de fetos, en el diámetro ánteroposterior (DAP) fue de $6,77 \mathrm{~mm}$ y el diámetro transversal (DT) de 9,23mm. Las mediciones efectuadas en el plano sagital determinaron que el espesor en la zona anterior (EZA) es de $1 \mathrm{~mm}$; en la zona media (EZM) de 0,59 $\mathrm{mm}$ y el espesor de la zona posterior (EZP) de 1,72 $\mathrm{mm}$. En el adulto, el promedio de las medidas del disco corresponden en el DAP a 14,46 mm y en el DT a 20,08 mm. Las dimensiones tomadas en el plano sagital señalan que el EZA es de 2,39mm; el EZM es 1,60mm y el EZP es 3,29 mm. Fue estadísticamente significativa la correlación de DT y DAP del disco articular en el feto $(\mathrm{p}=<0.005)$. En el disco adulto se determinó una correlación para EZA,EZM y EZP $(p=<0.005)$.

Las variaciones en las dimensiones del disco de la articulación témporomandibular pueden atribuirse al crecimiento de los elementos articulares, a la función masticatoria, a la presencia o ausencia de piezas dentarias, lo que se manifestaría en una modelación del espesor del disco, principalmente en la zona posterior.

PALABRAS CLAVE: Articulación témporomandibular; Disco; Morfometría.

\section{INTRODUCCIÓN}

La articulación témporomandibular humana (ATM), sinovial y bicondílea, une la mandíbula al hueso temporal, permitiendo la relación anatómica y funcional entre ambos. Participan en su constitución: el proceso condilar de la mandíbula, el tubérculo articular y la fosa mandibular del hueso temporal, a las cuales se agrega el disco articular, la cápsula, la sinovial y ligamentos extrínsecos e intrínsecos (Dubrul, 1991; Datamore \& Athanasiou, 2003).

Funcionalmente, la ATM permite que la mandíbula sea capaz de realizar movimientos de apertura y de cierre, además de movimientos de protusión, retrusión, lateralidad y combinación de todos ellos. Para efectuar esta dinámica, el proceso condilar realiza movimientos de rotación y translación gracias a la presencia de músculos y ligamentos asociados a las estructuras óseas y fibrosas (Sarnat \& Laskin, 1992).

Al nacer, la fosa mandibular es aplanada y poco profunda y existe escaso desarrollo del tubérculo articular. El proceso condilar de la mandíbula es plano. Durante los primeros meses de vida, el tubérculo temporal y la superficie articular del proceso condilar se mantienen planos, debido a que la principal actividad del sistema estomatognático es la succión. Cuando empieza la función masticatoria, el proce-

\footnotetext{
* Departamento de Ciencias Básicas, Facultad de Medicina, Universidad de La Frontera, Temuco, Chile.

** Departamento de Odontología Integral, Facultad de Medicina, Universidad de La Frontera, Temuco, Chile.

Financiado por Proyecto DIDUFRO 120426. Universidad de La Frontera, Temuco, Chile.
} 
so condilar mandibular se hace convexo y la fosa mandibular cada vez más cóncava. Además, se va engrosando el tubérculo articular (Wurgaft \& Montenegro, 2003). El crecimiento de la ATM se extiende hasta la segunda década de la vida post natal.

El disco articular es una estructura fibrosa que deriva del mesénquima, al igual que la cápsula de la ATM y el músculo pterigoideo lateral (Van der Linden \& Burdi, 1987). El disco articular presenta una forma cóncava-convexa en su cara superior y cóncava en su cara inferior, dividiendo a la articulación en dos unidades funcionalmente diferentes. El compartimento supradiscal es móvil, libre y deslizante, mientras que el infradiscal efectúa el movimiento de rotación. El disco articular participa en todos los movimientos y, además, puede realizar movimientos propios independientes de las estructuras óseas, gracias al tejido fibroso que lo constituye.

El disco articular se une a los polos del proceso condilar a través de los ligamentos colaterales, también llamados discales. El ligamento discal lateral une el extremo lateral del disco articular al polo lateral del proceso condilar. El ligamento discal medial une el extremo medial del disco articular al polo medial del proceso condilar (Okesson, 2003; Manns \& Díaz, 1983).

Al corte sagital, en el disco, en sentido ántero-posterior. se distinguen 4 zonas: zona anterior conformada por tejido fibroso denso; zona media o de trabajo, más delgada y de tejido fibroso denso sin vascularización ni inervación; zona posterior de tejido fibroso denso y zona retrodiscal o bilaminar de tejido conectivo laxo con fibras elásticas y colágenas muy vascularizado con abundantes fibras nerviosas (Okesson). De acuerdo a las observaciones de Montenegro \& Mery (1986), en la zona anterior del disco articular se insertan fibras musculares pertenecientes al músculo pterigoideo lateral; la zona media carece de vasos y nervios, al igual que la capa fibrosa o fibrocartílago que cubre las superficies articulares del proceso condilar y fosa mandibular, probablemente por el rol que cumplen al soportar grandes esfuerzos y compresión durante los movimientos articulares.

La presencia de capilares sanguíneos $(1,9 \%)$ es muy escasa en los discos articulares normales, encontrándose éstos en la periferia del disco articular, pero no se ha detectado la presencia de capilares linfáticos (Yoshida et al., 1999).

En relación a la forma del disco articular, en sentido sagital, el espesor de la zona posterior (EZP), central (EZM) y anterior (EZA), tienen una relación de 3:1:2 (Isberg, 2003), lo que significa que el disco articular es más grueso en su zona posterior, seguido por la anterior, siendo más delgado en la zona media (Okesson).
El espesor del disco articular en la zona media es de 0.2 a $1 \mathrm{~mm}$; aumenta progresivamente en grosor hacia la periferia y en dirección ántero-posterior, adquiriendo un espesor de 2 a $4 \mathrm{~mm}$ a nivel de la zona posterior y de 1 a $2 \mathrm{~mm}$ en la zona anterior (Ballesteros \& García, 1998; Guichet, 1977; Manns \& Díaz; Dubrul).

A los 4 meses del desarrollo intrauterino, el esbozo del disco articular está constituido por tejido mesenquimático condensado, ubicándose entre los compartimentos supra e infradiscal, distinguiéndose muy bien de los otros tejidos. En el recién nacido, el disco articular es aplanado y casi circular (Wurgaft \& Montenegro; Isberg).

Los objetivos de este trabajo fueron medir, comparar y analizar el tamaño del disco articular en adultos y fetos humanos, a fin de determinar la relación entre forma y tamaño, a través de la disección anatómica, complementada con un estudio morfométrico.

\section{MATERIAL Y MÉTODO}

El estudio se efectuó en 10 discos de ATM de adultos varones, desdentados parciales y totales, cuya edad promedio era 60 años, y 10 discos de ATM de fetos con más de 28 semanas de gestación, todos los cuales se extrajeron de cadáveres formolizados pertenecientes a la Unidad de Anatomía, Facultad de Medicina de la Universidad de La Frontera, Temuco, Chile.

Para extraer los discos articulares, se efectuó una disección, por planos, de las regiones temporal y maseterina, destinada a despejar la rama de la mandíbula y la ATM. Luego, se abrió la cápsula articular identificándose el disco articular en su posición anatómica.

Se resecó el proceso condilar a nivel del cuello mandibular, se seccionaron las fibras del músculo pterigoideo lateral que unían el disco articular a la cabeza superior de este músculo y se retiró el proceso condilar.

La pieza anatómica constituida por proceso condilar y el disco articular, se rotuló y guardó en un frasco con formol tamponado al $10 \%$. El procedimiento para extraer los discos articulares fue similar en adultos y fetos.

Para registrar la forma y aspecto de cada uno de los especímenes se efectuó un dibujo en sentido sagital y frontal de cada disco articular y, además, se fotografió cada uno de ellos a nivel de sus caras inferior, lateral, anterior y posterior. 
A continuación, se procedió a medir en cada uno de los discos de adulto y feto, con un caliper digital tipo Mitutoyo, los siguientes parámetros :

Diámetro ántero posterior (DAP): desde el punto mediano del margen anterior hasta el punto mediano del margen posterior.

Diámetro transversal (DT): desde el punto mediano del margen lateral hasta el punto mediano del margen medial.

Espesor zona anterior (EZA): medido en la parte media de la zona anterior

Espesor zona media (EZM ): medido en la parte central de la zona media del disco

Espesor zona posterior (EZP ): medido en la parte media de la zona posterior del disco.

Los datos fueron almacenados en planilla Access y analizados con programa estadístico Stata 8.0, usando el estadístico de prueba de Person para correlación de variables continuas, con un nivel de significancia estadística de 0.005 .

\section{RESULTADOS}

\section{Morfología de los discos articulares de la ATM en adul- tos y fetos.}

El disco articular de la ATM del adulto tiene una forma ovoídea, donde predomina el diámetro transversal sobre el ántero-posterior (Fig.1). En el feto, la forma del disco articular es ligeramente ovoídea, casi redondeada. (Fig. 2).

$\mathrm{Al}$ observar la cara superior (superficie craneal) del disco articular adulto, éste presenta una zona anterior marcada por un sobrerelieve discreto que da inserción a un fascículo de fibras musculares pertenecientes a la cabeza superior del músculo pterigoideo lateral (Fig. 3). Se continúa con la zona intermedia que es más delgada y, en algunos casos, casi translúcida.

En la región posterior aparece una marcada eminencia que se continúa con la zona retrodiscal, que presenta abundante cantidad de tejido conectivo, donde se distinguen vasos de gran calibre.

En cambio, bajo lupa, el disco articular de la ATM del feto, en su cara superior se observa casi plano, al no

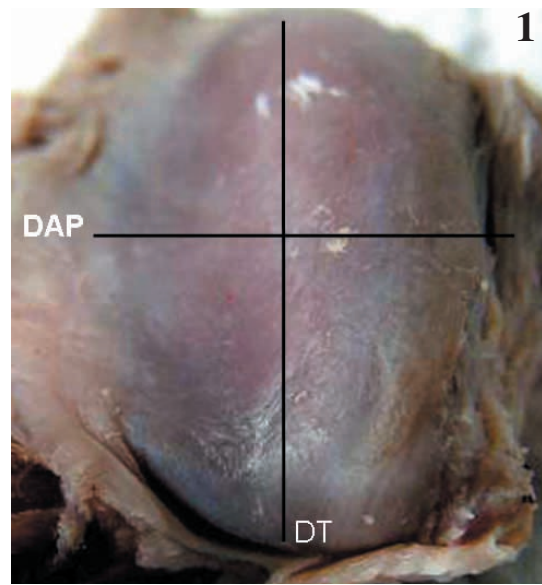

1
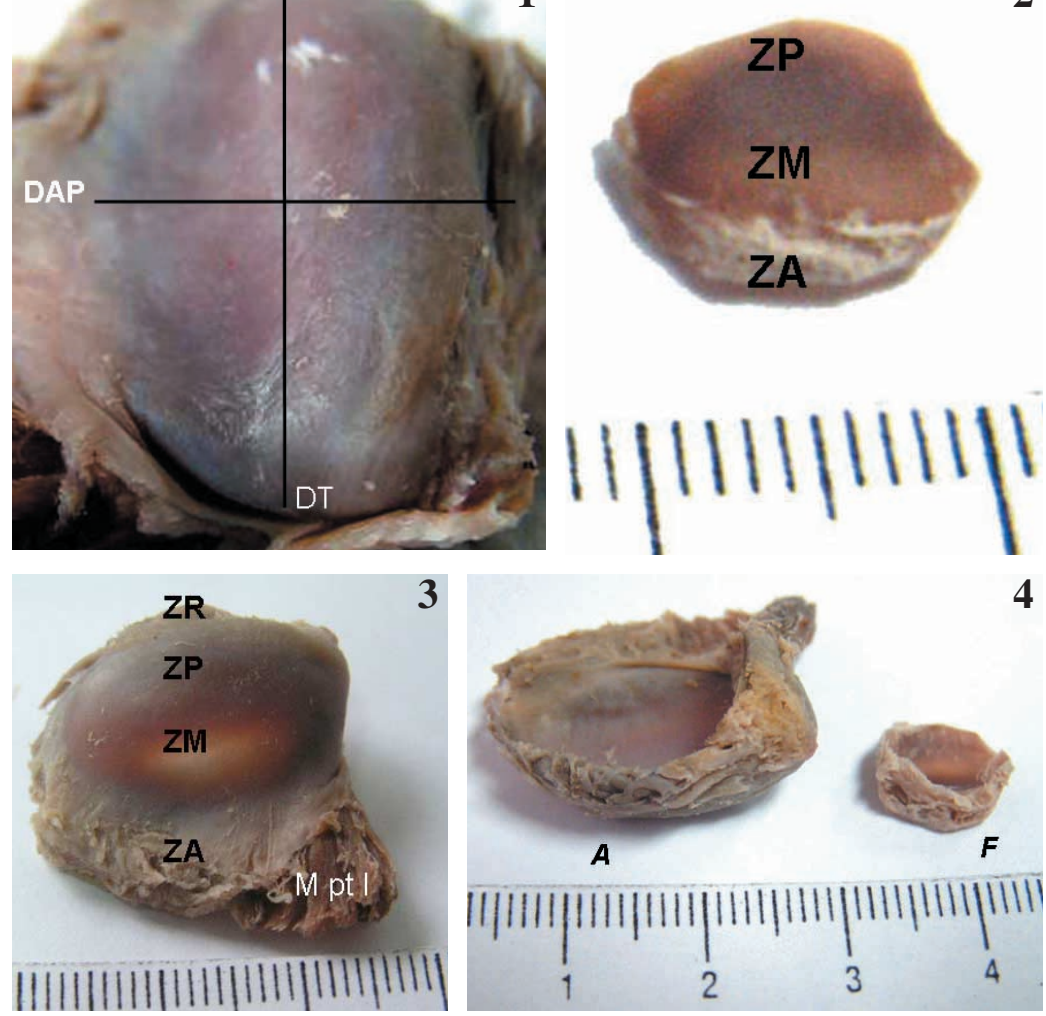

Fig. 1. Cara superior del disco articular de la articulación témporomandibular de un individuo adulto. ZA. Zona anterior; ZM. Zona media; ZP. Zona posterior; ZR. Zona retrodiscal.

Fig. 2. Cara superior del disco articular de la articulación témporomandibular de un feto. ZA. Zona anterior; ZM. Zona media; ZP. Zona posterior.

Fig. 3. Cara superior del disco articular de la articulación témporomandibular. DAP. Diámetro ántero-posterior. DT. Diámetro transversal.

Fig. 4. Cara inferior del disco articular de la articulación témporomandibular. 
apreciarse con nitidez los sobrerelieves anterior y posterior. La zona retrodiscal es muy pobre en tejido conectivo y, en algunos casos, casi no está presente. Además, las inserciones musculares en la zona anterior son muy escasas.

Al observar la cara inferior (superficie mandibular) del disco articular de adulto, se destaca notoriamente la concavidad correspondiente al proceso condilar de la mandíbula, mientras que en el disco articular de la ATM de feto esta concavidad o depresión es poco marcada (Fig. 4).

La cara lateral del disco articular del adulto tiene un aspecto de S itálica, con una zona anterior discretamente prominente y una zona posterior prominente y redondeada. Entre ambas se ubica la zona intermedia.

En el feto la cara lateral del disco articular de la ATM se observa como una superficie alargada y casi plana, con una ligera depresión en la parte central.

\section{Morfometría del disco articular de la ATM}

Con las mediciones efectuadas con el cáliper digital a los discos de fetos y adultosse obtuvieron los siguientes resultados:

En el feto, el promedio de las medidas del disco articular, corresponde en el DAP a 6,77mm (DS 1.43). El DT alcanzó a 9,23mm (DS 2.11). Las mediciones efectuadas en el plano sagital determinaron un promedio en el EZA de $0,82 \mathrm{~mm}$ (DS 0,40), el EZM de 0,59 mm (DS 0,57) y el EZP de 1,72 mm (DS 1,7).

En el adulto, el promedio de las medidas del disco articular, fueron: el DAP 14,46 mm (DS 1,1). El DT de 20,08 $\mathrm{mm}$ (DS 1,26). Las mediciones efectuadas en el plano sagital determinaron el promedio del EZA que fue de 2,39 mm (DS 1,44), el EZM de 1,60mm (DS 0,85 y el correspondiente a EZP de 3,29 mm (DS 1,02).

\section{Análisis estadístico de los resultados}

La correlación entre los diámetros transversal y ántero- posterior del disco articular de la ATM en fetos, dio un valor de $\mathrm{p}=0.0001$, lo cual es significativo. No existe esta correlación en los diámetros en los discos articulares de ATM de adultos, pues tienen un valor de $\mathrm{p}=0.450$, lo que no representa significancia estadística.

En cuanto al espesor en discos articulares de la ATM de fetos no se observa correlación en dos de las zonas estudiadas, dando valores $\mathrm{p}=0.2508$ entre las zonas posterior y media, $y$ de $p=0.484$ entre las zonas anterior y posterior. En tan- to, los valores entre las zonas media y anterior fue de $\mathrm{p}=0.050$ un valor límite, considerado significativamente estadístico.

En adultos, se observó significancia estadística entre todos los espesores, valor $\mathrm{p}=0.0067,0.0039$ y 0.0004 para las correlaciones entre las zonas posterior y anterior, posterior y media, y anterior, respectivamente.

\section{DISCUSIÓN}

En cuanto a la forma del disco articular de la ATM de adultos, las observaciones macroscópicas realizadas concuerdan con las descripciones de los autores consultados (Isberg; Wurgaft \& Montenegro; Detamore \& Athanasiou; Manns \& Diaz y Ballesteros \& García) reconociéndose 4 zonas bien marcadas: anterior, media, posterior y retrodiscal.

En el caso de la ATM de fetos, se constata un disco articular más aplanado y casi circular, en atención al poco desarrollo de las superficies articulares óseas vecinas. Es un poco diferente al del adulto, en el cual tiene forma ovoídea y corresponde a lo señalado por autores como Wurgaft \& Montenegro e Isberg.

El espesor de los discos articulares, tanto en fetos como en adultos, son concordantes con Okesson, quien describió que la zona posterior es la más gruesa, seguida de la zona anterior, siendo la zona media la más delgada.

Al comparar la proporcionalidad que describe Isberg de 3:1:2 para las zonas posterior, media y anterior, con las mediciones de este trabajo, se obtuvieron valores cercanos para los discos articulares de la ATM de adultos y fetos.

También se observa que el disco articular está unido a los polos del proceso condilar a través de estructuras ligamentosas, lo cual permite que el disco acompañe al proceso condilar en sus movimientos, tal como lo señala Manns \& Díaz y Okesson.

El hecho que el disco articular de la ATM del feto aparezca con menos desarrollo que el de adulto, en las zonas anterior y posterior, y con una zona retrodiscal muy escasa, podría indicar que estas partes del disco articular se desarrollan plenamente, cuando la persona comienza con la actividad masticatoria (Wurgaft \& Montenegro).

El promedio del espesor en la zona anterior del disco articular de ATM de adultos, fue de 2,38 mm, lo que es superior a lo reportado por Manns \& Díaz y Dubrul. Guichet reportó $2 \mathrm{~mm}$ y Ballesteros \& García 1,68mm. 
En la zona media del disco articular de la ATM de adultos, se encontró un valor de 1,60 mm, con una DS de O.85, lo que es concordante con Guichet, quien reportó 1-2 mm, y levemente superior a lo señalado por Dubrul quien indicó $1 \mathrm{~mm}$. Las mediciones de este trabajo son mayores a lo reportado por Ballesteros y García quienes indicaron un espesor de $0.7 \mathrm{~mm}$ y a lo reportado por Manns \& Díaz que fue de 0.2-0.4mm.

En la zona posterior, se encontró un valor de 3,29 mm con una DS de 1,02, lo que es concordante con lo reportado por Manns \& Díaz que fue de 3-4mm, y levemente diferente a lo obtenido por Dubrul y Guichet quienes midieron $3 \mathrm{~mm}$ de EZP. Por otra parte, Ballesteros \& García obtuvieron un espesor de 2,95 $\mathrm{mm}$ para EZP.

En la revisión bibliográfica no se encontraron antecedentes acerca del espesor del disco del feto en sus diferentes zonas, con el cual comparar las mediciones ejecutadas en la presente experiencia.

Los DAP del proceso condilar del adulto medidos por Ballesteros \& García, fueron de $10.29 \mathrm{~mm}$, lo que es menor que el DAP del disco del adulto de este trabajo $(14,46 \mathrm{~mm})$.

El DT es similar en el proceso condilar y disco articular de la ATM de adulto, pues se obtuvo un promedio de 20,08mm mientras que Ballesteros \& García encontraron un diámetro transversal del proceso condilar de 19,97mm.

Se requerirían mayores estudios para complementar la morfometría condilar y discal.
Al comparar las dimensiones de los discos articulares de las ATM de los fetos estudiados con las muestras de los adultos, en cuanto a sus diámetros DAP y DT, se observa la tendencia a mantener la proporcionalidad entre ambos diámetros. En los fetos, se observa un crecimiento y desarrollo progresivo en los diámetros mencionados, existiendo una correlación entre ellos. Esta correlación no se encontró en los adultos, a pesar que los DT, al igual que en fetos, son mayores que los DAP. Esto se puede deber al tamaño de la muestra y a la variabilidad de los sujetos observados.

En cuanto al porcentaje de crecimiento del EZA, EZM y EZP del disco articular de la ATM del feto y del adulto, se observa que existe una correlación entre los espesores de las zonas anterior, media y posterior de los discos articulares de adultos. Sin embargo, esta correlación sólo se observó en los fetos en los espesores medios y anteriores $(p=0.05)$.

Lo anterior podría sugerir que la zona posterior es la que más sufre de variaciones de tamaño durante su desarrollo y actividad funcional, pues esta situación es concordante con el hecho que un disco que tiende a ser aplanado en el feto, cambia su forma durante el crecimiento para hacer congruentes las superficies articulares (Wurgaft \& Montenegro e Isberg).

Finalmente se podría plantear, a la luz de estos resultados, que las variaciones en las dimensiones del disco articular de la ATM producen una modelación del disco en su espesor, principalmente en la zona posterior de éste.

MATAMALA, V. F.; FUENTES, F. R. \& CEBALLOS, C. M. Morphology and morphometry of the temporomandibular joint disc in human fetus and adults. Int. J. Morphol., 24(2):245-250, 2006.

SUMMARY: The human sinovial and bi-condile temporo mandibular joint (TMJ), is a set of structures that permit an anatomical and functional relationship between both temporary bone and jaw. This joint allows all the mandibular movements and functions of the stomatognatic system. One of the main elements of the TMJ is the articular disc, that divides the joint in two compartments and also relates the condile bone to the mandibular fossa, an the articular eminence of the temporary bone. In order to obtain morphometric data, 10 adults discs from 60 years average and 10 fetuses discs from more than 28 weeks of gestation, were analyzed and measured.

The articular dik from TMJ in adults agrees with those from literature and the fetus articular disk present a retrodiscal zone under developed. The anteroposterior diameter of the fetuses discs gave an average measurement of $6,77 \mathrm{~mm}$ and their transversal diameter was of $9,23 \mathrm{~mm}$. The sagital plane measurements determined an anterior zone thickness of $1 \mathrm{~mm}$, followed by a medial zone of $0,59 \mathrm{~mm}$. and a posterior zone thickness of $1,72 \mathrm{~mm}$. On the other side, an average of 14,46 $\mathrm{mm}$ were obtained on the antero-posterior adult dics diameter. The transversal or cross-sectional diameter registred here a measurement of 20,08 mm. The sagital plane measurements determined the anterior zone thickness as $2,39 \mathrm{~mm}$; the medial zone 1,60 mm and a posterior zone 3,29 mm. An important statistical significance was found in the correlationship between the anteroposterior and transversal or cross-section diameters in the fetus. In the adult instead, it was found a correlation between thicknesses of the anterior, medial and posterior zones.

Therefore, temporo-mandibular disc joint variations are attributable to anatomical elements growth, such as masticatory function and the presence or absence of dental pieces. We believed this situation would be mirrored in the modeling of disc thickness at the posterior zone.

KEY WORD: Temporomandibular joint; Disc; Morphometry. 


\section{REFERENCIAS BIBLIOGRÁFICAS}

Ballesteros, L. \& García, L. Morfometría de la articulación témporomandibular. Un estudio con material de autopsia. Medunab, 1(2):78-83, 1998.

Datamore, M. S. \& Athanasiou, K.A. Structure and function of the temporomandibular joint disc: implications for tissue engineering. J.Oral Maxillofac. Surg., 61(4):494506, 2003.

Dubrul, L. L. Anatomía oral de Sicher y Dubrul. $8^{\mathrm{a}}$ ed. São Paulo, Artes médicas, 1991.

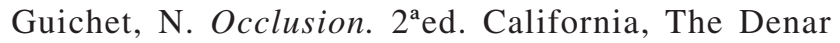
Corporation, 1977.

Isberg, Anika. Disfunción de la Articulación Temporomandibular. Ed. Artes Médicas Ltda. São Paulo, 2003.

Manns, A. \& Díaz, G. Sistema Estomatognático. Santiago, Empigraf, 1983.

Montenegro, M. A. Mery, \& C. Aguirre, A. Histología y Embriología del Sistema Estomatognático. Santiago, Ediciones Facultad de Odontología, Universidad de Chile, 1986.

Okesson, P. Tratamiento de Oclusión y Afecciones temporomandibulares. 5 ${ }^{\mathrm{a} e d}$. Elsevier, 2003.

Sarnat, B. \& Laskin, D. The temporomandibular joint: A biological basis for clinical pratice. $4^{\mathrm{a}}$ ed. Philadelphia, W. B. Saunders Company, 1992.

Van der Linden, E. J. \& Burdi, A. R. Critical periods in the prenatal morphogenesis of the human lateral pterygoid muscle, the mandibular condyle, the articular disk, and medial articular capsule. Am. J. Orthod. Dentofac. Orthop., 91:22-8, 1987.

Yoshida, H.; Fujita, S.; Nishida, M. \& Lizuka, T. Localization of lymph capillaries and blood capillaries in human temporomandibular joint discs. J. Oral Rehabil., 26(7):600-5. 1999.

Wurgaft, R. \& Montenegro, M. Desarrollo y estructura de la articulación temporomandibular. Santiago, Servimpres Ltda., 2003.
Dirección para correspondencia: Prof. Dr. Fernando Matamala Vargas Facultad de Medicina Universidad de La F Fontera Casilla 54-D

Temuco - CHILE

Email: matamala@ufro.cl

Recibido : 18-05-2005

Aceptado: 12-03-2006 